\title{
Lipidomics analysis of human follicular fluid form normal-weight patients with polycystic ovary syndrome: a pilot study
}

Yanna Ban ${ }^{1}$, Haiying Ran², Ying Chen ${ }^{1}$ and Li Ma ${ }^{3,4^{*}}$

\begin{abstract}
Background: The polycystic ovary syndrome (PCOS) is the most common endocrine associated with insulin resistance, even in the absence of overweight. The global lipid profile of the follicular fluid in PCOS with normal weight as yet has not been investigated. The objection of this pilot study was to explore the changes of lipids in the follicular fluid of PCOS with normal weight.
\end{abstract}

Methods: Follicular fluid samples were collected from patients who underwent IVF, including normal-weight women without PCOS (control group, $n=10$ ) and normal-weight women with PCOS (PCOS group, $n=8$ ). A lipidomic analysis was performed by high performance liquid chromatography/ mass spectrometry (HPLC-MS). Multidimensional statistical analysis was performed to disclose the global differences between the two groups. Further, differential lipid analysis between the two groups was performed by Fold Change Analysis (FC Analysis) and T-test to screen potential markers.

Results: All 812 species of 32 subclasses of lipids were identified by lipidomics analysis. 108 kinds of lipids were considered as the potential candidate differential metabolites with the score of variable importance in the project (VIP) more than 1 by the orthogonal partial least squares discriminant analysis. 32 lipids were significantly different between the PCOS group and the control group simultaneously with $\mathrm{FC}>1.5$ or $\mathrm{FC}<0.67, p$-value $<0.05$ and VIP value $>1$. These differential species of lipid belong to lipid subclasses including triglycerides (TG), phosphatidylethanolamines (PE) and phosphatidylinositols (PI).

Conclusion: The identified differential lipids in the follicular fluid may be considered as candidate biomarkers as well as therapeutic targets of PCOS with normal-weight.

Keywords: Polycystic ovary syndrome, Lipidomics, Follicular fluid, HPLC-MS

\section{Introduction}

Polycystic ovary syndrome (PCOS) is one of the most common endocrine and metabolic disorders with a prevalence ranging from 5 to $10 \%$ among women of reproductive age. The clinical manifestations include menstrual irregularities, anovulatory infertility, signs of androgen

*Correspondence: malicheng2002@163.com

${ }^{4}$ Laboratory of Lipid \&Glucose Metabolism, The First Affiliated Hospital of Chongqing Medical University, Chongqing 400016, P. R. China

Full list of author information is available at the end of the article excess, and metabolic and psychological disorders. POCS is the most important cause of anovulation infertility, with a high incidence of $18 \%$ [1]. In addition, the presence of low oocyte quality is common in PCOS patients undergoingin vitro fertilization (IVF) treatment [2]. Follicular fluid (FF) serves as acomplex microenvironment for oocyte growth, follicular maturation, and germ cell-somatic cell communication [1]. It accumulates all metabolisms during oocytes growth. Hence, the changes in the metabolites of FF have been linked to impaired oocyte quality and outcomes of IVF in patients with original author(s) and the source, provide a link to the Creative Commons licence, and indicate if changes were made. The images or other third party material in this article are included in the article's Creative Commons licence, unless indicated otherwise in a credit line to the material. If material is not included in the article's Creative Commons licence and your intended use is not permitted by statutory regulation or exceeds the permitted use, you will need to obtain permission directly from the copyright holder. To view a copy of this licence, visit http://creativecommons.org/licenses/by/4.0/. The Creative Commons Public Domain Dedication waiver (http://creativeco mmons.org/publicdomain/zero/1.0/) applies to the data made available in this article, unless otherwise stated in a credit line to the data. 
PCOS. Metabolomics is a high-throughput approach for the detection of extensive small-molecule metabolites in various biological samples that can provide useful information related to diagnostic biomarker and pathogenesis mechanism of diseases. Currently, metabolomics analysis of FF has revealed that lipid metabolites are significantly changed in PCOS patients [3, 4]. Lipidomics is one of the metabolomics approach that focuses on lipids and is a promising technique for overviewing lipid profiles in body fluid, blood and tissues. Currently, there are a few published lipidomics studies on PCOS in plasma $[5,6]$. However, the lipidomics of PCOS in FF, which directly reflects the oocyte micro-environment, has not been investigated previously.

Women with PCOS are often overweight or obese. However, due to the diversity of clinical and biochemical manifestations of PCOS, $30-50 \%$ of patients are with normal weight [7]. The presence or absence of obesity is one of the most important factors influencing PCOS phenotypes. The incidence of insulin resistance and metabolic syndrome was found to be higher in obese PCOS patients than in non-obese PCOS patients, but the latter also had metabolic abnormalities [8]. A previous study found that non-overweight women with PCOS had higher luteinizing hormone (LH) and follicle-stimulating hormone (FSH) levels in plasma than healthy women. Moreover, basal insulin, total cholesterol, low-density lipoprotein (LDL), very low-density lipoprotein (VLDL), and triglyceride levels in the overweight group were found to be significantly higher. However, there were no differences in triglyceride and total cholesterol in plasma between the normal normal-weight women with PCOS and normal-weight women without PCOS [9]. To date, the lipids in FF from normal-weight women with PCOS have never been studied. In the present pilot study, we used lipidomics technology based on high performance liquid chromatography/ mass spectrometry (HPLC-MS) platform to obtain a comprehensive picture of the lipid alterations that occur in the FF of PCOS patients with normal weight.

\section{Experimental \\ Subjects}

We designed a case-control pilot study, including FFs from 8 women with normal weight (body mass index $[\mathrm{BMI}]<25 \mathrm{~kg} / \mathrm{m}^{2}$ ) who were diagnosed with PCOS and 10 healthy women. This study was performed at the First Affiliated Hospital of Chongqing Medical University and wasconducted in accordance with the ethics guidelines of the Declaration of Helsinki. The FFs were collected from the patients who underwent IVF or intracytoplasmic sperm injection (ICSI) in our reproductive centre between January 2019 and December 2019. Written informed consents were obtained from all subjects, and all the experimental protocols were approved by the ethics committee of the First Affiliated Hospital of Chongqing Medical University. The enrolment criteria for PCOS were based on the presence of any two or three features - hyperandrogenism, menstrual irregularity, and polycystic ovary morphology, according to the Rotterdam consensus criteria. None of the women had abnormal serum androgen hormone levels. The control group included women who sought treatment for tubal infertility or male factors with normal ovarian reserve (regular menstrual cycles, and anti-Müllerian hormone [AMH] concentration of $\geq 1.1 \mathrm{ng} / \mathrm{mL}$ ) and normal BMI. Women with endometriosis, glucose metabolism, cancer, or other medical disorders that could affect folliculogenesis were excluded. The gonadotropin-releasing hormone antagonist protocol was used for controlled ovarian stimulation. When the follicle reached an average diameter of $\geq 18 \mathrm{~mm}$, urinary human chorionic gonadotropin (u-HCG, Lizhu, Zhuhai, China) was administered, and ultrasound-guided FF samples were collected after $36 \mathrm{~h}$ using an 18-gauge single-lumen aspiration needle.

\section{Preparation of samples}

Serum and plasma samples were collected between days 2 and 4 of the menstrual cycle in the control group and between 2 and 4 days after a spontaneous bleeding episode in patients with PCOS after an overnight fast. Circulating levels of hormones were measured by electrochemiluminescence assay (Cobas e602; Roche Diagnostics GmbH, Mannheim, Germany) and the biochemical indexes were measured by enzymatic method with an automatic biochemical analyzer (Cobas c701; Roche Diagnostics GmbH, Mannheim, Germany). Clear FF samples without macroscopic blood contamination were included. After oocyte isolation, the pooled FF samples were centrifuged at $800 \times \mathrm{g}$ for $10 \mathrm{~min}$ to remove particulates. The FF supernatant was stored at $-80^{\circ} \mathrm{C}$ until analysis. Before analysis, the FF samples were thawed in a $4^{\circ} \mathrm{C}$ water bath. Lipids were extracted using methyl tert-butyl ether (MTBE) method. Briefly, the samples were homogenized with $200 \mu \mathrm{L}$ of water and $240 \mu \mathrm{L}$ of methanol. Then $800 \mu \mathrm{L}$ of MTBE was added and the mixture was ultrasound $20 \mathrm{~min}$ at $4{ }^{\circ} \mathrm{C}$ followed by sitting for $30 \mathrm{~min}$ at room temperature. The solution was centrifuged at $14,000 \times \mathrm{g}$ for $15 \mathrm{~min}$ at $10^{\circ} \mathrm{C}$ and the upper organic solvent layer was obtained and dried under nitrogen. The samples were reconstituted in $200 \mu \mathrm{L}$ of $90 \%$ isopropanol/acetonitrile and centrifuged at $14,000 \times \mathrm{g}$ for $15 \mathrm{~min}$ at $10^{\circ} \mathrm{C}$, and $3 \mu \mathrm{L}$ of the sample was injected into the LC-MS system. A pooled FF sample from all healthy controls and PCOS patients was used as quality 
control $(\mathrm{QC})$ and underwent the same sample extraction procedures.

\section{Chemicals and reagents}

MS-grade methanol, MS-grade acetonitrile and HPLCgrade isopropanol were purchased from ThermoFisher (Thermo Fisher Scientific Co., Waltham, Massachusetts, USA). HPLC-grade formic acid, HPLC-grade ammonium formate and MTBE were purchased from Sigma-Aldrich (Sigma Chemical Co., St. Louis, Missouri, USA).

\section{UPLC-MS experiments}

The UPLC-MS experiments were performed using a Q-Exactive Plus mass spectrometer (Thermo Scientific) system equipped with a UHPLC Nexera LC-30A (SHIMADZU). Mass spectrometry performed in either the ESI positive or negative mode. ESI parameters were optimized and preset for all measurements as follows: Source temperature, $300^{\circ} \mathrm{C}$, Capillary Temp, $350^{\circ} \mathrm{C}$, the ion spray voltage was set at $3000 \mathrm{~V}$, S-Lens RF Level was set at $50 \%$ and the scan range of the instruments was set at m/z 200-1800. Reversed phase chromatographic separation was performed on a Waters ACQUITY UPLC CSH C18 column $(2.1 \mathrm{~mm} \times 100 \mathrm{~mm}, 1.7 \mu \mathrm{m})$ maintained at $45^{\circ} \mathrm{C}$. Solvent A was acetonitrile-water (6:4, $\mathrm{v} / \mathrm{v}$ ) with $0.1 \%$ formic acid and $0.1 \mathrm{mM}$ ammonium formate and solvent B was acetonitrile-isopropanol (1:9, $\mathrm{v} / \mathrm{v}$ ) with $0.1 \%$ formic acid and $0.1 \mathrm{mM}$ ammonium formate. The initial mobile phase was $30 \%$ solvent B at a flow rate of $300 \mu \mathrm{L} / \mathrm{min}$. It was held for $2 \mathrm{~min}$, and then linearly increased to $100 \%$ solvent B in $23 \mathrm{~min}$, followed by equilibration with $5 \%$ solvent $B$ for $10 \mathrm{~min}$.

The samples were analyzed in a random order. To test the reproducibility of the sample preparation procedure and LC-MS analysis, QC samples were injected at the beginning of the run and after every eight real samples. Each of the prepared QC samples was analyzed only once.

\section{Data processing}

The raw data were processed by LipidSearch software version 4.1 (Thermo Scientific $^{\mathrm{TM}}$ ) for peak identification, lipid identification by MS/MS, peak extraction, peak alignment, and quantitative analysis. The parameters were set as follows: precursor tolerance: $5 \mathrm{ppm}$, product tolerance: $5 \mathrm{ppm}$, and product ion threshold: $5 \%$. The extraction data was normalized by total peak area after removed the lipid with RSD > 30\% among samples.

\section{Statistical analysis}

The software simca-p 14.1 (Umetrics, Umea, Sweden) was used for pattern recognition. After pareto-scaling pretreatment, multidimensional statistical analysis was performed, including unsupervised principal component analysis (PCA) and orthogonal partial least square discriminant analysis (OPLS-DA). One-dimensional statistical analysis by SPSS Statistics v17.0.0 (SPSS, Inc., Chicago, USA) included Student's t-test and variance multiple analysis. $\mathrm{R}$ software was used to draw the volcano map, hierarchical clustering analysis map and correlation analysis map. Comparisons of parameters between two groups were performed by independent-Sample $\mathrm{T}$ test or non-parametric test, depending on the distribution (normal or not) of the examined variables.

\section{Results}

\section{Clinical characteristics}

The selected clinical baseline characteristics ofthe participants in our study are summarized in Table 1 . The patients and controls were well matched for age and BMI. There were no significant differences in LH, FSH, LH/ FSH, E2 (Estradiol), T (testosterone) levels, blood glucose, cholesterol, triglyceride, high-density lipoprotein cholesterol (HDL-C), low-density lipoprotein cholesterol (LDL-C), fertilization rate, cleavage rate and embryo topquality rate between normal-weight PCOS patients and

Table 1 Main clinical characteristics of the study groups

\begin{tabular}{llll}
\hline & Controls $(\boldsymbol{n}=$ ten) & PCOS( $\boldsymbol{n}$ =eight) & $\boldsymbol{P}$-value \\
\hline Age (years) & $28.80 \pm 2.74$ & $27.12 \pm 3.40$ & 0.26 \\
$\mathrm{BMI}\left(\mathrm{kg} / \mathrm{m}^{2}\right)$ & $20.96 \pm 1.99$ & $19.96 \pm 1.77$ & 0.28 \\
$\mathrm{LH}(\mathrm{mlU} / \mathrm{mL})$ & $4.20 \pm 2.61$ & $5.57 \pm 2.48$ & 0.22 \\
$\mathrm{FSH}(\mathrm{mlU} / \mathrm{mL})$ & $5.86 \pm 1.64$ & $6.66 \pm 2.21$ & 0.39 \\
$\mathrm{LH} / \mathrm{FSH}$ & $0.76 \pm 0.63$ & $0.93 \pm 0.49$ & 0.28 \\
$\mathrm{E}_{2}(\mathrm{pg} / \mathrm{mL})$ & $44.3 \pm 40.69$ & $68.75 \pm 39.91$ & 0.22 \\
$\mathrm{~T}(\mathrm{ng} / \mathrm{mL})$ & $0.49 \pm 0.14$ & $0.53 \pm 0.11$ & 0.52 \\
Glucose $(\mathrm{mmol} / \mathrm{l})$ & $4.83 \pm 0.42$ & $5.19 \pm 0.46$ & 0.52 \\
Cholesterol (mmol/L) & $4.25 \pm 0.25$ & $4.12 \pm 0.32$ & 0.77 \\
triglyceride (mmol/L) & $0.87 \pm 0.28$ & $0.88 \pm 0.40$ & 0.96 \\
HDL-C (mmol/L) & $1.47 \pm 0.28$ & $1.56 \pm 0.54$ & 0.64 \\
LDL-C (mmol/L) & $2.40 \pm 0.71$ & $2.02 \pm 0.41$ & 0.17 \\
AMH (ng/mL) & $3.52 \pm 1.12$ & $12.97 \pm 3.42$ & $<0.001^{*}$ \\
No.of Oocyte retrieve & $13.2 \pm 5.59$ & $22.3 \pm 5.99$ & $0.004^{*}$ \\
Normal fertilization & $87.03 \pm 0.18$ & $79.24 \pm 0.16$ & 0.40 \\
Rate(\%) & & & \\
Cleavage Rate(\%) & $95.08 \pm 0.57$ & $87.76 \pm 0.11$ & 0.07 \\
Embryo top-quality & $43.44 \pm 0.21$ & $53.42 \pm 0.27$ & 0.43 \\
Rate(\%) & & & \\
\hline
\end{tabular}

*is indicated $p<0.05$ values. Data presented as mean \pm standard deviation (SD) Abbreviations: $B M I$ body mass index; $F S H$ follicle-stimulating hormone; $L H$ luteinizing hormone; $E 2$ estradiol; $T$ testosterone; $H D L-C$ high-density lipoprotein cholesterol; $L D L-C$ low-density lipoprotein cholesterol; $A M H$ anti-Mullerian hormone

normal fertilization rate $=$ no. oocytes with $2 \mathrm{PN}$ and $2 \mathrm{~PB} /$ no. $\mathrm{COC}$ inseminated $\times 100 \%$; cleavage rate $=$ no. cleaved embryos on Day $2 /$ no. 2 PN/2PB oocytes on Day $1 \times 100 \%$; embryo top-quality rate = top quality embryos $/$ no. normally fertilized oocytes $\times 100 \%$. 
controls. AMH and No. of oocyte retrieve in PCOS were significantly higher than the control group.

\section{Quality control}

Four QC samples were analyzed by both ESI+ and ESIscan models, described in Section 2.4. Next, the base peak chromatograms were extracted and overlapped to compare the response intensity and the retention time of the peak. The results showed that the response intensity and retention time of the peak of each QC sample basically overlapped (shown in Figure 1 of the supplementary material). Pearson correlation analysis was then applied to the four QC samples of the response intensity of the extracted peaks. The results showed that the correlation coefficients were higher than 0.9 as shown in Figure 2 of the supplementary material. The peaks of all experimental samples and QC samples were extracted to perform PCA after Pareto-scaling. The results showed that the QC samples were closely packed together. A multivariate control chart was used to monitor the stability of the method. The Y-axis of the chart is the variance of the first principal component of the PCA model for all QC samples, and the $\mathrm{X}$-axis is the loading order of the QC samples. The variance was no more than two standard deviations, indicating that the analysis system was stable and all the data were under control (Fig. 1). The relative standard deviation (RSD) of peaks in the QC samples is an important indicator of data quality. The ratio of peaks with an RSD of no more than $30 \%$ was more than $80 \%$ in the four QC samples. All these results indicated that the method had good repeatability, reliability, and stability for lipidomics analysis.

\section{Identification of lipid compounds}

Data obtained from the positive and negative scan models of HPLC-MS were analyzed by LipidSearch. All 812 species of 32 lipid subclasses were identified (Fig. 2). Thedifferences between the two groups in lipid subclasses are shown in figure 3 of the supplementary material.

\section{Data modeling}

In this study, the PCA of FF was constructed to reveal the global differences of lipid between PCOS patients and healthy controls. In the PCA model, the clusters were not distinctly separated in the score plots, which might be due to the complexity and variation of clinical samples (shown in figure 3 of the supplementary material). The green box represents healthy controls, and the blue dot represents PCOS patients. OPLS-DA is a supervised method developed from PCA to remove unrelated noise from the

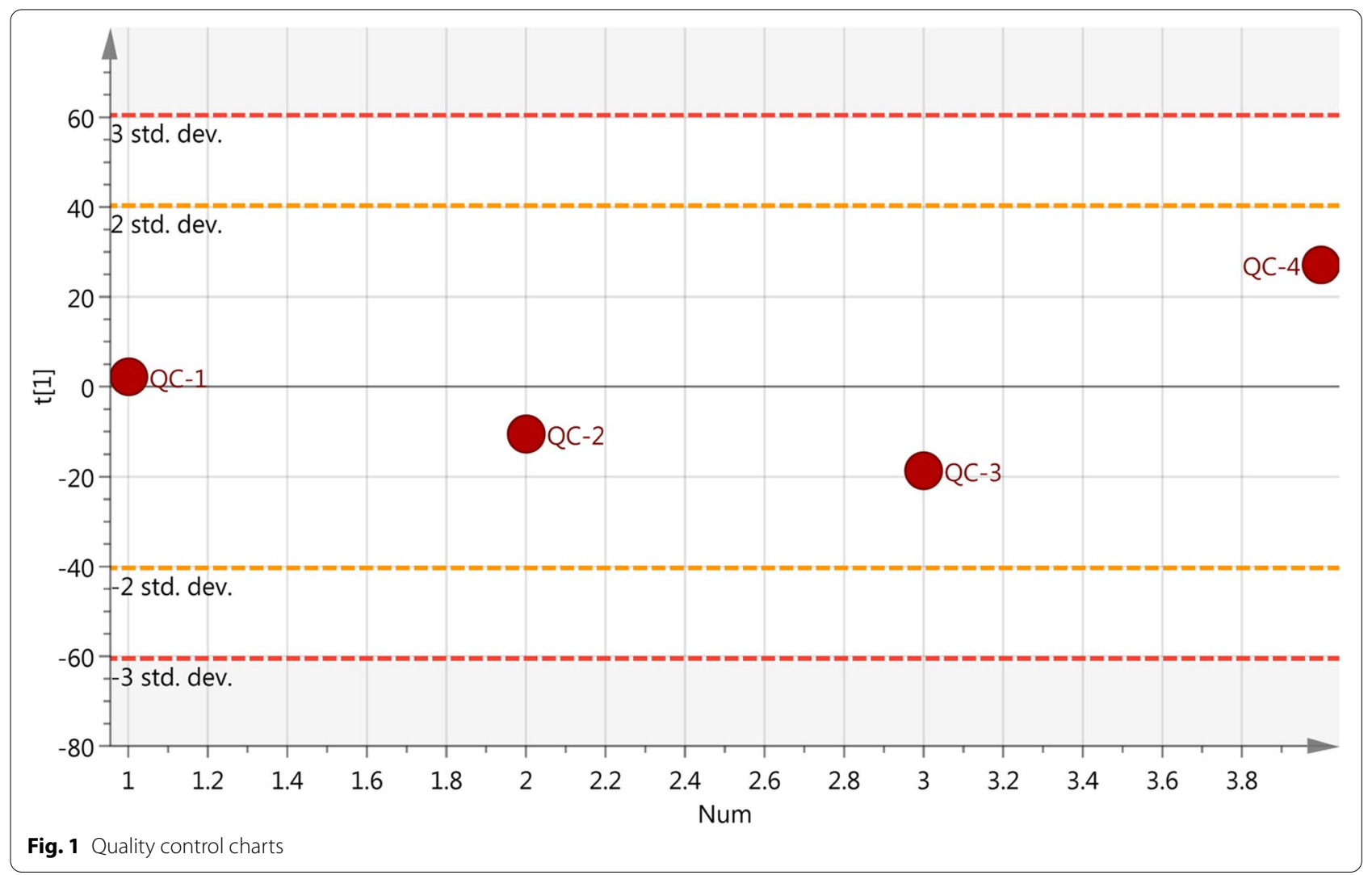




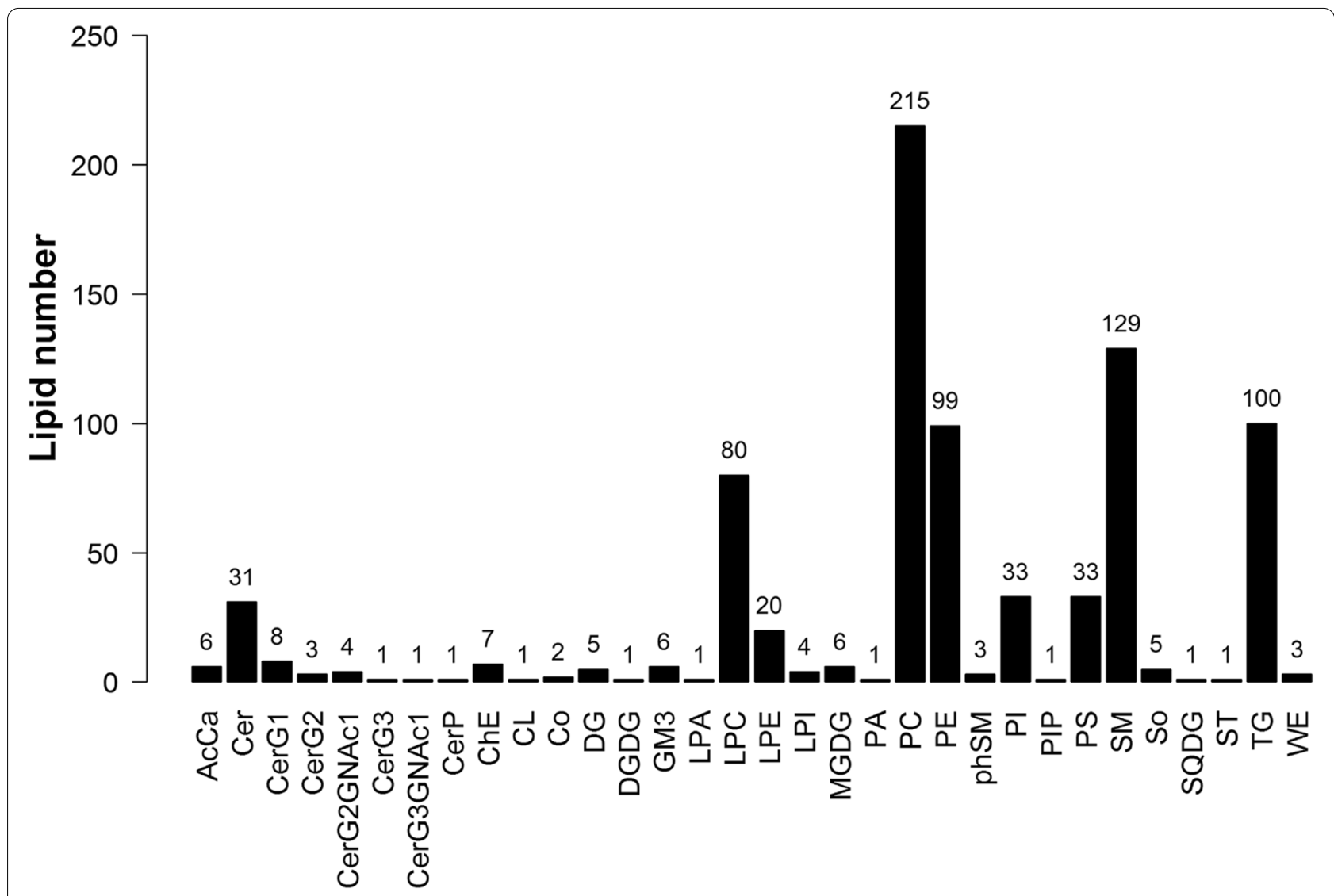

Fig. 2 Classification of the identified lipids

predictive variables and obtain more reliable metabolite information. The two-dimensional scores of the OPLSDA plot showed a clear separation between the healthy controls and PCOS patients with $\mathrm{p}(\mathrm{CV}-\mathrm{ANOVA})=2.3 \mathrm{E}-$ 005. (Fig. 3).

\section{Identification of potential biomarkers}

Potential differential metabolites were defined according to the variable importance in projection (VIP) values based on OPLS-DA. Usually, lipid molecules with VIP $\geq 1$ are considered to have contribute significantly to $\mathrm{c}$ model interpretation. As a result, 108 typesof lipids were considered as potential candidate differential metabolites with VIP scores more than 1. Among these lipids, 36 were significantly different between the PCOS group and the control group analyzed by T-test/non-parametric test $(P<0.05)$, as shown in Table 1 of the supplementary material. The differential lipids between the two groups were performed by Fold Change Analysis (FC Analysis) and T-test to screen potential biomarkers. The screening criteria is $\mathrm{FC}>1.5$ or $\mathrm{FC}<0.67$ and $P$ value $<0.05$. The information of the differential expression multiples, $P$ values and VIP values of lipid molecules were displayed in a volcano plot, as shown in Fig. 4. The purple dots in the volcano plot are the differential lipid molecules that are simultaneously with $\mathrm{FC}>1.5, P$ value $<0.05$ and VIP value $>1$.The area of the dots represents the VIP valve. The larger the bubble area shown in the plot, the greater the VIP value. As a result, there are 32 lipids with FC $>1.5$, P value $<0.05$ and VIP value $>1$ simultaneously Table 2).

\section{Discussion}

PCOS is a highly heterogeneous disease. In women with PCOS, the oocytes are often weak quality, which leads to lower fertilization, cleavage, implantation, and increased miscarriage rates [10]. The oocyte microenvironment provides the necessary requirements for oocyte developmental competence. FF is the liquid that surrounds the oocyte, forms its micro-environment and plays a key role in its development [3]. Therefore, it is crucial to explore the FF metabolite pattern. Untargeted metabolomics, which focuses on the dynamic changes of all small molecules in response to the disturbance of the organism, can provide deep insights for the etiopathogenesis and the discovery of biomarkers for various 


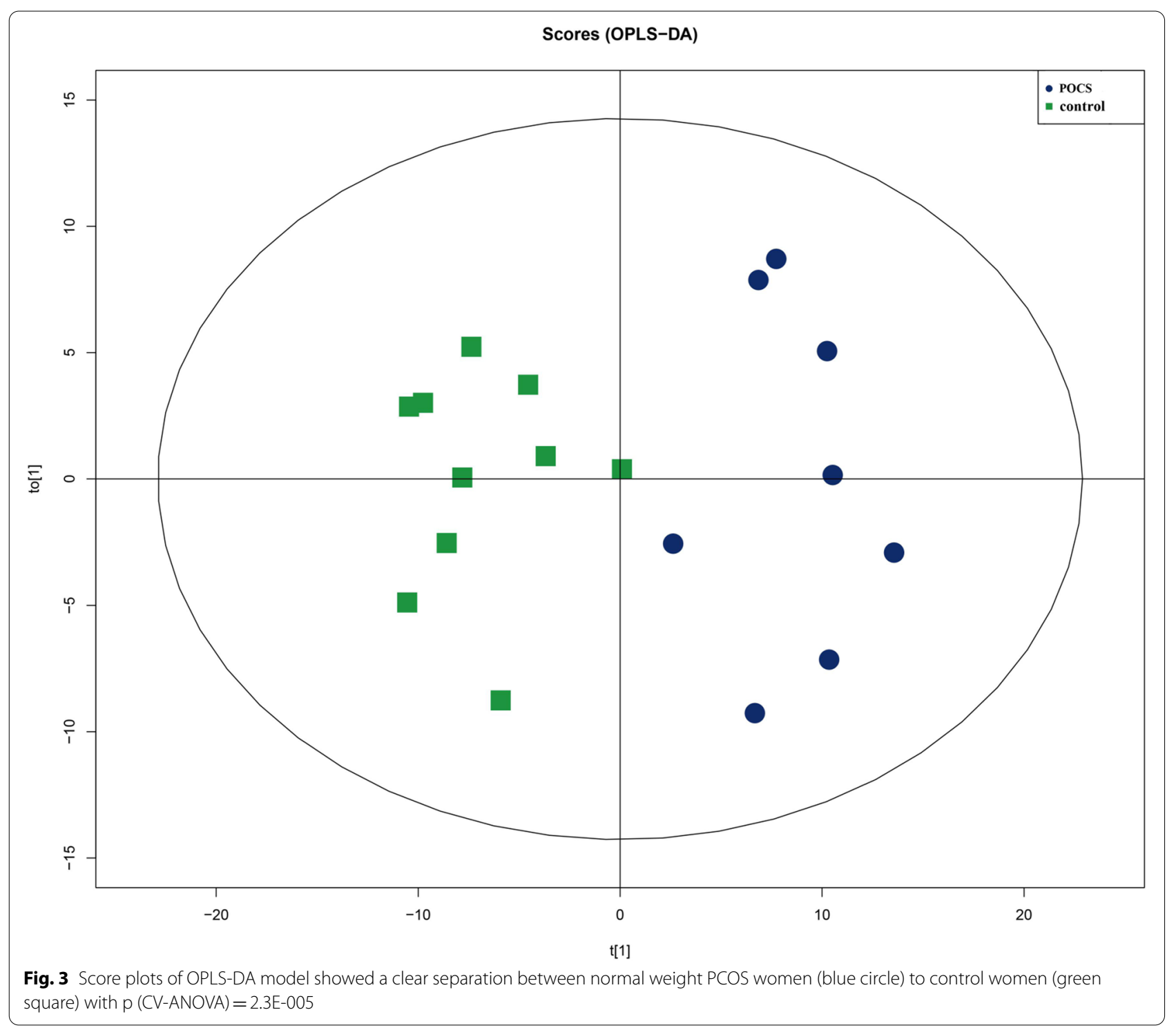

diseases [11]. Several researches of metabolomics on PCOS in plasma have found that the lipids were significantly changed. Lipidomics, a branch of metabolomics, is a high-throughput analytical technique that can systematically and efficiently analyze lipid composition and expression changes in various biological processes. At present, lipidomics analysis technology is generally based on liquid chromatography-mass spectrometry (LC-MS) platform and is mainly divided into untargeted and targeted analysis. Non-targeted lipidomics can systematically analysis of various types of lipids in the sample without bias, and the targeted lipidomics is mainly used for selective and specific quantitative analysis of specific lipids [12].
The present pilot study is the first to comprehensively investigate lipid profile changes in PCOS with normal weight in FF using the untargeted lipidomics approach based on ultra-high performance LC coupled with Q-Exactive MS. A number of lipids were detected in FF by this sensitive technique. We identified a series of differential lipids including triglycerides (TG), phosphatidylethanolamines (PE), phosphatidylinositols (PI) and etc. between women with PCOS and the healthy women.

TG was the only differentially represented subclass of lipids in FF of PCOS patients and health controls in this study (shown in Figure 4 of supplementary material). TG is a combination of three fatty acids combined with glycerol whichis the main source of energy. Patients with PCOS often have dyslipidemia mainly 


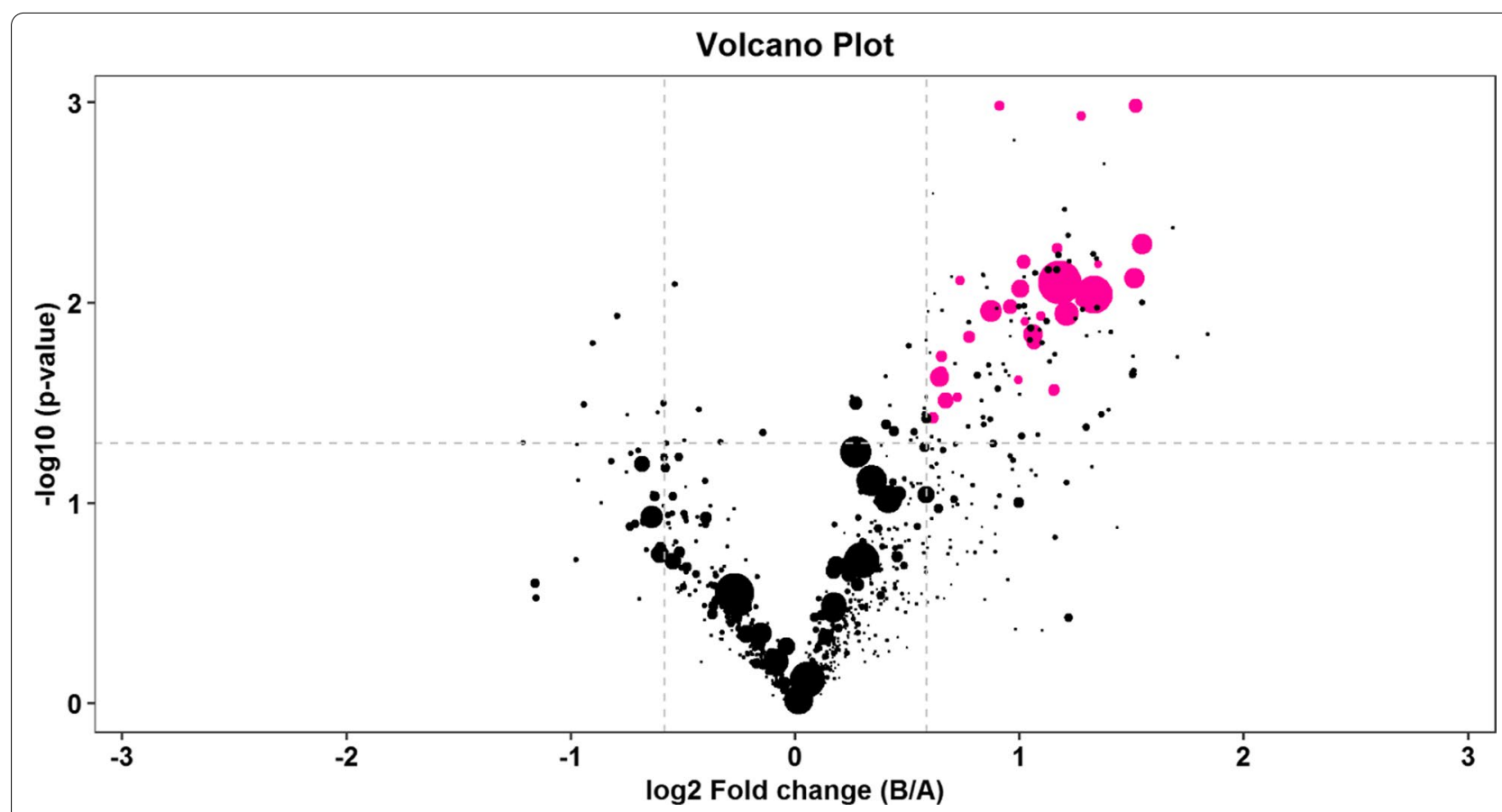

Fig. 4 The volcano plot based on FC, VIP and $P$ values of the differential lipid molecules. The purple dots represent the differential lipid molecules with $F C>1.5, P$ value $<0.05$ and VIP value $>1$ simultaneously. The area of the dots represents the VIP valve

includes high levels of LDL and TG and low levels of HDL. Furthermore, lipid abnormalities are closely associated with obesity, insulin resistance and hyperandrogenemia in PCOS patients [13]. On the other hand, obesity has an important influence on the lipid metabolism [14]. Recently, a case-control study was conducted on 153 women with PCOS and 449 healthy women as controls to compare the serum lipid profile. Each group was divided into normal, overweight and obesity subgroups according to the BMI. Surprisingly, significant differences in TG were found only between obese women with PCOS and obese women without PCOS. There was no difference in plasma TG levels between the two groups of non-obesity women [15]. As refer to FF, Liu et al. found that a reduced level of TG was highly related to the lower fertilization rate in PCOS [16]. However, increased BMI is associated with elevated TG levels in ovarian FF [17]. This research on the lipid profile in FF was enrolled PCOS women with average weight heavier than normal women. To our knowledge, the lipid metabolite profiles in FF of PCOS patients and normal women with weight matched were not been investigated previously. In this study, significantly increased TG levels in FF were found in PCOS compared with normal women. Increased TG levels might be associated with low quality of oocyte in PCOS patients. A research found that mouse cumulus-oocyte complexes exposed to lipid-rich FF during their maturation had increased oocyte lipid content, induction of endoplasmic reticulum stress markers, and impaired oocyte nuclear maturation [17]. TG accumulation in the FF was also correlated to the levels of adipokines and proinflammatory cytokines in FF, implying inflammatory processes in the FF that are caused by high TG levels and may also attenuate oocyte development [18].

In this study, many species of PE showed higher accumulation in FF of the PCOS patients. Considering the potential functions of $\mathrm{PE}$, it was found that $\mathrm{PE}$ is a major phospholipid class in the membranes of eukaryotic cells and modulates the membrane fluidity [19]. FF was centrifuged at $800 \mathrm{~g}$ which only pellet cells were removed but not organelles and vesicles. So the determined PE and PI may come from membrane structures of sub organelles or vesicles. PE also have been determined in FF by other lipidomic analysis. FF samples collected from patients who underwent IVF, including normal responder women who became pregnant (control group), women with PCOS and a hyper response to gonadotropins (PCOS group) and women with only hyper response to gonadotropins (HR group) were found that some form of PEs were higher represented HR groups and lower represented in PCOS group than the control group [20]. Regarding our study, although many forms of PE were high presented in PCOS patients, the total PE level was 
Table 2 The candidate lipids which as potential biomarkers to separate normal weight PCOS women to control women

\begin{tabular}{|c|c|c|c|c|c|c|c|}
\hline Lipidlon & Class & Ion Formula & CalMz & RT-(min) & Fold Change & $P$-value & VIP \\
\hline$P E(16: 0 / 22: 6)+H$ & $P E$ & C43 H75 O8 N1 P1 & 764.522484 & 10.2352508 & 2.1364297 & 0.01170255 & 1.2827738 \\
\hline $\mathrm{TG}(16: 0 / 14: 0 / 18: 1)+\mathrm{NH} 4$ & TG & $\mathrm{C} 51 \mathrm{H} 100 \mathrm{O} 6 \mathrm{~N} 1$ & 822.754516 & 20.7435549 & 2.82786013 & 0.00768395 & 1.04024184 \\
\hline $\mathrm{TG}(16: 1 / 16: 1 / 18: 1)+\mathrm{NH} 4$ & TG & $\mathrm{C} 53 \mathrm{H} 100 \mathrm{O} 6 \mathrm{~N} 1$ & 846.754516 & 19.714067 & 2.85935032 & 0.00103754 & 2.07802736 \\
\hline $\mathrm{TG}(16: 0 / 16: 0 / 18: 2)+\mathrm{NH} 4$ & TG & $\mathrm{C} 53 \mathrm{H} 102 \mathrm{O} 6 \mathrm{~N} 1$ & 848.770166 & 20.8812114 & 2.84645308 & 0.00753119 & 3.11342089 \\
\hline $\mathrm{TG}(16: 0 / 16: 0 / 18: 1)+\mathrm{NH} 4$ & TG & $\mathrm{C} 53 \mathrm{H} 104 \mathrm{O} 6 \mathrm{~N} 1$ & 850.785816 & 21.7834094 & 2.9164798 & 0.00508461 & 3.14637718 \\
\hline $\mathrm{TG}(16: 1 / 18: 1 / 18: 2)+\mathrm{NH} 4$ & TG & $\mathrm{C} 55 \mathrm{H} 102 \mathrm{O} 6 \mathrm{~N} 1$ & 872.770166 & 19.859873 & 2.51669633 & 0.00941933 & 4.83457578 \\
\hline $\mathrm{TG}(16: 0 / 18: 1 / 18: 2)+\mathrm{NH} 4$ & $\mathrm{TG}$ & $\mathrm{C} 55 \mathrm{H} 104 \mathrm{O} 6 \mathrm{~N} 1$ & 874.785816 & 20.8954062 & 2.26683859 & 0.00796182 & 7.04217237 \\
\hline $\mathrm{TG}(16: 0 / 18: 1 / 18: 2)+\mathrm{NH} 4$ & TG & $\mathrm{C} 55 \mathrm{H} 104 \mathrm{O} 6 \mathrm{~N} 1$ & 874.785816 & 19.8521076 & 2.42105627 & 0.00117044 & 1.21618584 \\
\hline $\mathrm{TG}(16: 0 / 18: 1 / 18: 1)+\mathrm{NH} 4$ & TG & $\mathrm{C} 55 \mathrm{H} 106 \mathrm{O} 6 \mathrm{~N} 1$ & 876.801466 & 21.7866945 & 2.51369634 & 0.00910841 & 6.11421082 \\
\hline $\mathrm{TG}(18: 0 / 16: 0 / 18: 1)+\mathrm{NH} 4$ & TG & $\mathrm{C} 55 \mathrm{H} 108 \mathrm{O} 6 \mathrm{~N} 1$ & 878.817116 & 22.584629 & 2.22068302 & 0.0275356 & 1.3333522 \\
\hline $\mathrm{TG}(18: 1 / 18: 2 / 18: 3)+\mathrm{NH} 4$ & TG & C57 H102 O6 N1 & 896.770166 & 19.4825832 & 2.55170831 & 0.00642044 & 1.03093598 \\
\hline $\mathrm{TG}(18: 2 / 18: 2 / 18: 2)+\mathrm{NH} 4$ & TG & $\mathrm{C} 57 \mathrm{H} 102 \mathrm{O} 6 \mathrm{~N} 1$ & 896.770166 & 18.8771548 & 2.24487784 & 0.00535694 & 1.51541463 \\
\hline $\mathrm{TG}(16: 0 / 18: 1 / 20: 4)+\mathrm{NH} 4$ & TG & C57 H104 O6 N1 & 898.785816 & 20.5420673 & 2.42182935 & 0.00972723 & 1.68243907 \\
\hline $\mathrm{TG}(18: 1 / 18: 2 / 18: 2)+\mathrm{NH} 4$ & TG & $\mathrm{C} 57 \mathrm{H} 104 \mathrm{O} 6 \mathrm{~N} 1$ & 898.785816 & 19.884727 & 2.00402129 & 0.00849049 & 2.6606902 \\
\hline $\mathrm{TG}(18: 1 / 18: 1 / 18: 2)+\mathrm{NH} 4$ & TG & C57 H106 O6 N1 & 900.801466 & 20.9160682 & 1.83056246 & 0.01101498 & 3.33870883 \\
\hline $\mathrm{TG}(18: 0 / 18: 1 / 18: 2)+\mathrm{NH} 4$ & TG & $\mathrm{C} 57 \mathrm{H} 108 \mathrm{O} 6 \mathrm{~N} 1$ & 902.817116 & 20.8947311 & 1.52937154 & 0.03751919 & 1.62092888 \\
\hline $\mathrm{TG}(18: 0 / 18: 1 / 18: 2)+\mathrm{NH} 4$ & TG & $\mathrm{C} 57 \mathrm{H} 108 \mathrm{O} 6 \mathrm{~N} 1$ & 902.817116 & 21.8017114 & 2.30938518 & 0.01129426 & 3.83179623 \\
\hline $\mathrm{TG}(16: 0 / 18: 1 / 20: 1)+\mathrm{NH} 4$ & TG & $\mathrm{C} 57 \mathrm{H} 110 \mathrm{O} 6 \mathrm{~N} 1$ & 904.832766 & 22.5836836 & 2.22447076 & 0.02719304 & 1.57736708 \\
\hline $\operatorname{Cer}(\mathrm{d} 18: 1 / 16: 0)+\mathrm{HCOO}$ & Cer & C 35 H68 O5 N1 & 582.510298 & 11.3804774 & 1.65187002 & 0.02982499 & 1.19827027 \\
\hline $\mathrm{PE}(16: 0 / 18: 2)-\mathrm{H}$ & PE & C39 H73 O8 N1 P1 & 714.507931 & 10.7122963 & 2.02509893 & 0.00623164 & 2.06847352 \\
\hline $\mathrm{PE}(16: 0 / 18: 1)-\mathrm{H}$ & $P E$ & C39 H75 O8 N1 P1 & 716.523581 & 11.4653225 & 1.87622225 & 0.00103417 & 1.36389091 \\
\hline PE(16:0/20:4)-H & $P E$ & C41 H73 O8 N1 P1 & 738.507931 & 10.5345784 & 1.94530888 & 0.01049121 & 2.08653155 \\
\hline PE(18:0/18:2)-H & $P E$ & C41 H77 O8 N1 P1 & 742.539231 & 11.6753321 & 1.59339478 & 0.03091878 & 2.34017355 \\
\hline PE(18:0/18:1)-H & $P E$ & C41 H79 O8 N1 P1 & 744.554881 & 12.413823 & 1.66288434 & 0.00777766 & 1.2000378 \\
\hline $\mathrm{PE}(16: 0 / 22: 6)-\mathrm{H}$ & $P E$ & C43 H73 O8 N1 P1 & 762.507931 & 10.2584914 & 2.08517498 & 0.01442886 & 3.08345178 \\
\hline PE(18:1/20:4)-H & $P E$ & C43 H75 O8 N1 P1 & 764.523581 & 10.6214751 & 1.71023624 & 0.0148648 & 1.68604552 \\
\hline $\mathrm{PE}(16: 0 / 22: 5)-\mathrm{H}$ & PE & C43 H75 O8 N1 P1 & 764.523581 & 10.9153492 & 2.03051326 & 0.01237958 & 1.17476918 \\
\hline PE(18:0/20:4)-H & PE & C43 H77 O8 N1 P1 & 766.539231 & 11.5051781 & 1.55899537 & 0.02358573 & 2.91621258 \\
\hline $\mathrm{PE}(18: 1 / 22: 6)-\mathrm{H}$ & PE & C45 H75 O8 N1 P1 & 788.523581 & 10.323258 & 1.98966059 & 0.02424517 & 1.17590614 \\
\hline $\mathrm{PE}(18: 0 / 22: 6)-\mathrm{H}$ & PE & C45 H77 O8 N1 P1 & 790.539231 & 11.2313382 & 2.08883459 & 0.01561633 & 2.20426914 \\
\hline $\mathrm{PI}(16: 0 / 18: 2)-\mathrm{H}$ & $\mathrm{Pl}$ & $\mathrm{C} 43 \mathrm{H} 78 \mathrm{O} 13 \mathrm{~N} 0 \mathrm{P} 1$ & 833.518557 & 9.29326139 & 1.56851322 & 0.01842781 & 1.53643448 \\
\hline $\mathrm{PI}(16: 0 / 18: 1)-\mathrm{H}$ & $\mathrm{Pl}$ & C43 H80 O13 N0 P1 & 835.534207 & 10.0911401 & 1.56876413 & 0.0223933 & 1.66545385 \\
\hline
\end{tabular}

found no significant difference between the two groups in this paper.

Finally, phosphatidylinositol (PI) was found to be presented in high levels in the PCOS patients with normal weight. PI is composed of a glycerol backbone, two acyl chains esterified and an inositol ring linked by a phosphate. Although PI constitutes only $5-10 \%$ of total cellular lipids in mammalian cells, it is the source of seven phosphorylated derivatives of PI which play a major role in a vast array of cellular functions including signalling, membrane trafficking, ion channel regulation and actin dynamics [21]. The PI subclass of lipid has previously been detected in FF. Thais et al. compared MALDI-MS lipid fingerprints in the FF of young poor responder women in and normal responders. A lipid ion belonging to the PI subclass was overrepresented in the poor ovarian response group [22]. Another lipidomic analysis of FF samples revealed that some PIs were higher in normal responder women than in women with PCOS and a hyper response to gonadotropins and women with only hyper response to gonadotropins. In this study, some forms of PI were high presented in PCOS patients, but the total PI level was found no significant difference between the two groups.

In this pilot study, lipid alterations of FF were found in PCOS patients with normal-weight, and those lipids might be considered as potential biomarkers of oocyte micro-environment in PCOS patients with normalweight. However, there are some limitations. The results were obtained using a small set of samples because strict 
criteria were used to screen the patients enrolled in this pilot study. This experimental design was a biomarker discovery study in general and the sample was used as a screening set, and hence the prospective biomarkers proposed in this study need to be confirmed in an independent cohort as the validation set. This study revealed that the lipid profiles of normal-weight women with PCOS were different from those found in normal women. Furthermore, the potential lipid markers found in FF, highlighted by the relative increase in TG in the PCOS groups, contributed to improving the understanding of the molecular mechanisms involved in PCOS women without overweight. These biomarkers have demonstrated that the lipids are related to molecular processes in the normal-weight PCOS, such as inflammatory processes and endoplasmic reticulum stress in FF, which are caused by high TG levels and may impaired oocyte nuclear maturation. For this reason, PCOS patients always have the impaired oocyte development during ART. Therefore, FF lipid profile analysis is an important tool for identifying a panel of potential biomarkers because it reflects the ovarian microenvironment.

\section{Conclusions}

In conclusion, this is the first study using the untargeted lipidomics technology based on HPLC-MS to analyze the lipid subclasses alterations in normal-weight PCOS focusing on the micro-environment of the oocyte. A pattern recognition technique allowed us to specifically discriminate normal-weight patients with from the normal women during IVF, which described a comprehensive picture of the lipid alterations that occurred in PCOS. The identified dysfunctional lipids of TG, PE and PI might serve as important diagnostic tools and are closely related to alteration in FF of PCOS with normal weight.

\begin{abstract}
Abbreviations
POCS: Polycystic ovary syndrome; IVF: In vitro fertilization; COC: Cumulusoocyte complexes; FF: Follicular fluid; BMI: Body mass index; ICSI: Intracytoplasmic sperm injection; FSH: Follicle-stimulating hormone; LH: Luteinizing hormone; $\mathrm{E}_{2}$ : Estradiol; T: Testosterone; HDL-C: High-density lipoprotein cholesterol; LDL-C: Low-density lipoprotein cholesterol; AMH: Anti-Mullerian hormone; LDL: Low-density lipoprotein; HDL: High-density lipoprotein; TG: Triglycerides; PE: Phosphatidylethanolamines; PI: phosphatidylinositols.
\end{abstract}

\section{Supplementary Information}

The online version contains supplementary material available at https://doi. org/10.1186/s13048-021-00885-y.

Additional file 1.

Acknowledgements

Not applicable.

\section{Authors' contributions}

YNB and LM contributed to the conception and design of the study; YNB, HYR and $Y C$ consulted the literature; $Y N B$ and $Y C$ enrolled the patients and collected the sample; LM performed the data acquisition and statistical analysis; YNB, HYR and YC wrote the first draft of the manuscript. LM contributed to the manuscript revision. The authors read and approved the submitted version.

\section{Funding}

This work was supported by grants from the National Nature Science Foundation of China [31700725 to Li Ma]; the Natural Science Foundation of Chongqing [2020jcyj-msxmX0504 to Li Ma]; and the Joint Medical Research Project of Chongqing Health Commission [2019MSXM060 to Ying Chen].

\section{Availability of data and materials}

The datasets used and/or analysed during the current study are available from the corresponding author upon reasonable request.

\section{Declarations}

Ethics approval and consent to participate

This study was approved by the First Affiliated Hospital of Chongqing Medical University and all human biologic materials were collected after receiving written informed consent from patients.

\section{Consent for publication}

Not applicable.

\section{Competing interests}

The authors declare that they have no competing interests.

\section{Author details}

${ }^{1}$ Reproductive Medicine Center, The First Affiliated Hospital of Chongqing Medical University, Chongqing 400016, P. R. China. ${ }^{2}$ Biomedical Analysis Center, College of Basic Medical Sciences, Army Medical University, Chongging 400038, P. R. China. ${ }^{3}$ The Chongqing Key Laboratory of Translational Medicine in Major Metabolic Diseases, The First Affiliated Hospital of Chongqing Medical University, Chongqing 400016, P. R. China. ${ }^{4}$ Laboratory of Lipid \&Glucose Metabolism, The First Affiliated Hospital of Chongqing Medical University, Chongqing 400016, P. R. China.

Received: 3 February 2021 Accepted: 20 September 2021

Published online: 13 October 2021

\section{References}

1. Zhang X, Xu X, Li P, et al. TMT Based Proteomic Analysis of Human Follicular Fluid From Overweight/Obese and Normal-Weight Patients With Polycystic Ovary Syndrome. Front Endocrinol (Lausanne). 2019;10:821.

2. Dehghan Z, Mohammadi-Yeganeh S, Salehi M. MiRNA-155 regulates cumulus cells function, oocyte maturation, and blastocyst formation. Biol Reprod. 2020;103(3):548-59.

3. Chen X, Lu T, Wang X, et al. Metabolic alterations associated with polycystic ovary syndrome: a UPLC Q-Exactive based metabolomic study. Clin Chim Acta. 2020:502:280-6.

4. Sun ZG, Chang HM, Wang AJ, et al. Identification of potential metabolic biomarkers of polycystic ovary syndrome in follicular fluid by SWATH mass spectrometry. Repro bio endocrinol. 2019;17(1):45.

5. Haoula Z, Ravipati S, Steke DJ, et al. Lipidomic analysis of plasma samples from women with polycystic ovary syndrome. Metabolomics. 2015;11(3):657-66.

6. Moran $L J$, Mundra PA, Teede $\mathrm{HJ}$, et al. The association of the lipidomic profile with features of polycystic ovary syndrome. J Mol Endocrinol. 2017:59(1):93-104.

7. Gonzalez F. Nutrient-induced inflammation in polycystic ovary syndrome: role in the development of metabolic aberration and ovarian dysfunction. Semin Reprod Med. 2015;33(4):276-86.

8. Romano LG, Bedoschi G, Melo AS, et al. Metabolic abnormalities in polycystic ovary syndrome women: obese and non obese. Rev Bras Ginecol e Obstet. 2011;33(6):310-6. 
9. Tugrul S, Kutlu T, Pekin O, et al. Clinical, endocrine, and metabolic effects of acarbose, a alpha-glucosidase inhibitor, in overweight and nonoverweight patients with polycystic ovarian syndrome. Fertil Steril. 2008;90(4):1144-8.

10. Fraison E, Kostova E, Moran $L$, et al. Metformin versus the combined oral contraceptive pill for hirsutism, acne, and menstrual pattern in polycystic ovary syndrome. Cochrane Database Syst Rev. 2020;13(8):CD005552.

11. Ma $L$, Zhang $X Q$, Pan F, et al. Urinary metabolomic analysis of intrahepatic cholestasis of pregnancy based on high performance liquid chromatography/mass spectrometry. Clin Chim Acta. 2017;71:292-7.

12. Alshehry ZH, Mundra PA, Barlow CK, et al. Plasma Lipidomic profiles improve on traditional risk factors for the prediction of cardiovascular events in type 2 diabetes mellitus. Circulation. 2016;134(21):1637-50.

13. Zhu JL, Chen Z, Feng WJ, et al. Sex hormone-binding globulin and polycystic ovary syndrome. Clin Chim Acta. 2019;499:142-8.

14. Hussain A, Alam JM. Dyslipidaemia in woman with polycystic ovarian syndrome: a case control study in tertiary care hospital of Karachi. J Pak Med Assoc. 2014;64(9):1049-52.

15. Rashidi H. Serum lipid profile and insulin resistance in women with polycystic ovary syndrome (PCOS). J Diabetes Metab Disord. 2018;5(3):107-11.

16. Liu LY, Yin TL, Chen Y, et al. Follicular dynamics of glycerophospholipid and sphingolipid metabolisms in polycystic ovary syndrome patients. J Steroid Biochem Mol Biol. 2019;185:142-9.
17. Yang $X, W u L L, C$ chura $L R$, et al. Exposure to lipid-rich follicular fluid is associated with endoplasmic reticulum stress and impaired oocyte maturation in cumulus-oocyte complexes. Fertil Steril. 2012;97(6):1438-43.

18. Batushansky A, Zacharia A, Shehadeh A, et al. A shift in Glycerolipid metabolism defines the follicular fluid of IVF patients with unexplained infertility. Biomolecules. 2020;10(8):1135.

19. Dawaliby R, Trubbia C, Delporte C, et al. Phosphatidylethanolamine is a key regulator of membrane fluidity in eukaryotic cells. J Biol Chem. 2016;291(7):3658-67.

20. Cordeiro FB, Cataldi TR. L. do Vale Teixeira da Costa, et al. follicular fluid lipid fingerprinting from women with PCOS and hyper response during IVF treatment. J Assist Reprod Genet. 2015;32(1):45-54.

21. Blunsom NJ, Cockcroft S. Phosphatidylinositol synthesis at the endoplasmic reticulum. Bba-Mol Cell Biol. 1865;2020(1):158471.

22. Cataldi T, Cordeiro FB, Costa Ldo V, et al. Lipid profiling of follicular fluid from women undergoing IVF: young poor ovarian responders versus normal responders. Hum Fertil. 2013;16(4):269-77.

\section{Publisher's Note}

Springer Nature remains neutral with regard to jurisdictional claims in published maps and institutional affiliations.
Ready to submit your research? Choose BMC and benefit from:

- fast, convenient online submission

- thorough peer review by experienced researchers in your field

- rapid publication on acceptance

- support for research data, including large and complex data types

- gold Open Access which fosters wider collaboration and increased citations

- maximum visibility for your research: over $100 \mathrm{M}$ website views per year

At BMC, research is always in progress.

Learn more biomedcentral.com/submissions 